\title{
On Weakly Analytic and Faithfully Convex Functions in Convex Programming*
}

\section{Garth P. McCormick $\dagger$ Christoph Witzgall}

†Emeritus, Department of Operations Research, SEAS

The George Washington University Washington, DC 20052

‡U.S. DEPARTMENT OF COMMERCE Technology Administration Mathematical and Computational Sciences Division National Institute of Standards and Technology

Gaithersburg, NiD 20899 



\section{On Weakly Analytic and Faithfully Convex Functions in Convex Programming*}

\section{Garth P. McCormick $\dagger$ Christoph Witzgallł}

†Emeritus, Department of Operations Research, SEAS

The George Washington University

Washington, DC 20052

‡U.S. DEPARTMENT OF COMMERCE Technology Administration Mathematical and Computational Sciences Division

National Institute of Standards and Technology

Gaithersburg, MD 20899

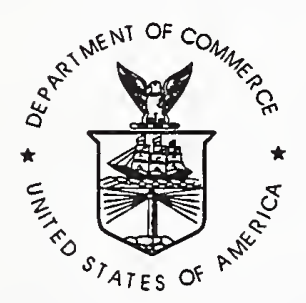

U.S. DEPARTMENT OF COMMERCE William M. Daley, Secretary

TECHNOLOGY ADMINISTRATION Dr. Cheryl L. Shavers, Under Secretary of Commerce for Technology

NATIONAL INSTITUTE OF STANDARDS AND TECHNOLOGY

Raymond G. Kammer, Director

* Contribution of the National Institute of Standards and Technology (NIST) and not subject to copyright in the United States. Research supported in part by: NSF Grant ECS-8709795, ONR Grant N00014-89J1537, ONR Contract N-0014-87-F0053. 
Abstract Weakly-analytic convex, faithfully convex, and self-concordant functions are considered, and their relationships described. The purpose of the report is to state and provide complete proofs of results that illustrate circumstances under which weak analyticity occurs for convex functions.

Keywords: convex functions, convex programming, faithfully convex, interior point methods, self-concordant, SUMT, weakly analytic

\section{Introduction}

In the course of examining convex programming problems and their duality properties, Rockafellar [5] introduced the class of "faithfully convex" functions, a class comprising convex functions which are analytic in $R^{n}$, including convex quadratic and linear functions. A more general class of functions, called, "weakly analytic" functions is described in the monograph by Bank et al. [1] on parametric optimization. The term alludes to the fact that analytic functions are also weakly analytic when defined over an entire space $R^{n}$.

Convex programming problems, if their defining functions involve weakly analytic functions, exhibit special properties of their SUMT limits, in particular, convergence to analytic centers of the optimality region as shown in [3].

What does the definition of "weakly analytic" imply? What classes of functions are weakly analytic? Is there some kind of characterization of such functions? Any analysis of that kind will have to emphasize the multi-variable case, because functions of a single real variable are weakly analytic simply if they are constant on the entire line of real numbers or else exhibit no constant intervals.

In analogy to the known result that faithfully convex functions are the direct sums of linear and strictly convex functions, we show that convex weakly analytic functions are the direct sums of constants and functions without constant intervals.

Faithfully convex functions are trivially weakly analytic. Another class of convex functions - fundamental to the performance analysis of the widely applied "interior point methods" for linear and nonlinear constrained optimization - is the class of "self-concordant" functions introduced by Nesterov and Nemirovskii (e.g. [4]). If defined over an entire space $R^{n}$, self-concordant functions are also faithfully convex, as noted by the aforementioned authors. Those functions are, therefore, also weakly analytic. We will also describe a proof of this proposition based on providing a differential inequality which implies faithful convexity. 

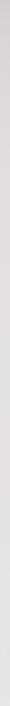
Most material of this report is not new. Similar results are generally known. They are, however, scattered through the literature, and proofs are often merely sketched because of their essentially elementary nature. Also, the material of this report has been referred to without proof in the forthcoming journal article [3] by the authors. The report is thus intended to provide back-up information for that article.

\section{Weakly Analytic Convex Functions}

(2.1) Definition: A function $c(x)$ defined on $C \subseteq R^{n}$ is

$$
\text { "weakly analytic" [1] }
$$

if for any line $L$ and any line segment $S=[\underline{x}, \bar{x}] \subset L, \underline{x} \neq \bar{x}$, the following holds: if the function $c(x)$ is constant on the segment $S$, then it is defined on the entire line $L$, i.e. $L \subseteq C$, and is constant on that line.

Functions which are analytic on $R^{n}$ are plainly weakly analytic in their full region of analyticity. This includes linear and quadratic functions as well as multivariate polynomials.

Analytic functions which do not cover the entire space $R^{n}$ may not be weakly analytic as, for instance, the function

$$
f(x, y)=\frac{y}{x}, \quad x>0,
$$

or even the convex analytic function

$$
c(x, y)=\frac{x+y}{2}-\sqrt{x y}, \quad x>0, y>0,
$$

which vanishes along the ray $\{(x, y): x=y, x>0, y>0\}$ but not along the entire line containing the ray.

Because of the following well-known fact, the concept of weakly analytic functions is particularly fruitful if those functions are also convex or concave. Indeed (e.g. $[6],[8])$, 

(2.2) if a convex (concave) function $c(x)$ is defined on an open convex set $K$, and is defined and constant on a line $L$, then it is defined and constant on any parallel line through a point $x^{\prime}$ in $K$. In other words, if $w \neq 0$ is a vector in the direction of line $L$, then

$$
c(x+\theta w)=c(x) \text {, for any } x \in K \text { and } \theta \in R \text {. }
$$

Proof: Denote by $L^{\prime} \neq L$ the line through $x^{\prime}$ parallel to $L$. Then choose any $x^{\prime \prime} \in L$. Since $K$ is open, there exists $x^{(0)} \in K$ such that the point $x^{\prime} \in L^{\prime}$ lies on the line segment spanned by $x^{(0)}$ and $x^{\prime \prime}$. Then there are coefficients $\lambda, \mu>0, \lambda+\mu=1$, such that

$$
x^{\prime}=\lambda x^{(0)}+\mu x^{\prime \prime} .
$$

For each point $x^{(1)} \in L^{\prime}$ consider the the point $x^{(2)} \in L$ which is the intersection of the line through $x^{(0)}$ and $x^{(1)}$ with the line $L^{\prime}$. That intersection exists, since The lines $L$ and $L^{\prime}$ are parallel. The latter also implies that

$$
x^{(1)}=\lambda x^{(0)}+\mu x^{(2)}
$$

with the same coefficients $\lambda, \mu$ as above. By convexity, $x^{(1)} \in K$ and, because $x^{(1)}$ is in general position, $L^{\prime} \subset K$. Furthermore, in the case of a convex function $c(x)$,

$$
c\left(x^{(1)}\right) \leq \lambda c\left(x^{(0)}\right)+\mu c\left(x^{(2)}\right) .
$$

Since $x^{(0)}$ is fixed, and $c\left(x^{(2)}\right)=c\left(x^{\prime \prime}\right)$ is constant, $c\left(x^{(1)}\right)$ is bounded above on line $L^{\prime}$. By convexity, $c(x)$ must therefore be constant on that line. The case of a concave function $c(x)$ is handled analogously.

Following is a direct consequence of (2.2).

(2.3) If a convex (concave) function $c(x)$ defined on an open convex set $K$ is weakly analytic (2.1) then the following holds: If $c(x)$ is constant on some line segment $S=[\underline{x}, \bar{x}]$, then every line $L$ parallel to $S$ either lies entirely outside $K$ or is fully contained by it. In the latter case, $c(x)$ is constant on $L$.

We proceed to characterize convex (concave) weakly analytic functions. 

(2.4) Definition: We call a function $Q(s)$

\section{"constance-free"}

if $Q(s)$ is not constant on any line segment $S=[\underline{s}, \bar{s}], \underline{s} \neq \bar{s}$.

Constance-free functions are, of course, weakly analytic, as are constant functions. We will also restrict our dicussion to the convex case since the concave case is analogous.

(2.5) Theorem: $A$ convex function $c(x)$ on an open convex set $K \subseteq R^{n}$ is weakly analytic if and only if it is the direct sum of a constant function and a constance-free convex function. In other words, a convex function $c(x)$ is weakly analytic if and only if it is of the form

$$
c(x)=Q(A x),
$$

where $A$ denotes an $\ell \times n-m a t r i x$, and the function

$$
Q(s), s \in A K=\{A x: x \in K\} \subseteq R^{\ell},
$$

is convex and constance-free.

Proof. Suppose the convex function $c(x)$ is weakly analytic, and let $D$ be the set of all directions $w$ in which $c(x)$ is constant along a line. The key observation here is that $D$ is a linear subspace of $R^{n}$. Since any multiple of $u$ and $v$ is in $D$, it suffices to show that $u+v \in D$ or, equivalently, that $w=(u+v) / 2 \in D$. By convexity and a simple rearrangement of terms,

$$
\begin{aligned}
c(x+\theta w) & =c\left[\frac{1}{2}(x+\theta u)+\frac{1}{2}(x+\theta v)\right] \\
& \leq \frac{1}{2} c(x+\theta u)+\frac{1}{2} c(x+\theta v)=c(x),
\end{aligned}
$$

which shows that $c(x+\theta w)$ is bounded above for all $\theta \in R$ and therefore constant, again by convexity.

Thus $D$ may be described as the null-space of an $\ell \times n$-matrix $A$ of full rank $\ell$ : $D=\{w: A w=0\}$. By $D^{\perp}$ we denote the linear subspace which is spanned by the colums of the matrix $A^{T}$, and which forms the orthogonal complement to $D$. Any $x \in R^{n}$ is split uniquely, in familiar fashion,

$$
x=\left[I-A^{T}\left(A A^{T}\right)^{-1} A\right] x+A^{T}\left(A A^{T}\right)^{-1} A x=w+\hat{x},
$$



where it is readily seen that

$$
w=\left[I-A^{T}\left(A A^{T}\right)^{-1} A\right] x \in D \text { and } \hat{x}=A^{T}\left(A A^{T}\right)^{-1} A x \in D^{\perp} .
$$

We then find

$$
c(x)=c(w+\hat{x})=c(\hat{x})=c\left[A^{T}\left(A A^{T}\right)^{-1} A x\right]=Q(A x) .
$$

The function

$$
Q(s)=c\left[A^{T}\left(A A^{T}\right)^{-1} s\right], s \in A K \subseteq R^{\ell}
$$

is convex since it is the composition of a linear function with a convex function. We proceed to show that it is constance-free. Assume to the contrary that $\underline{s} \neq \bar{s}$ define a constant line segment of $Q(s)$. Then $\underline{x}=A^{T}\left(A A^{T}\right)^{-1} \underline{s}$ and $\bar{x}=A^{T}\left(A A^{T}\right)^{-1} \bar{s}$ are points in $R^{n}$ with $\underline{s}=A \underline{x}$ and $\bar{s}=A \bar{x}$. It follows that the points $\underline{x}, \bar{x}$ bracket a constant line segment $S$ for $c(x)$. Since $c(x)$ is weakly analytic, it is then constant everywhere in direction $w=\bar{x}-\underline{x}$, which thus must lie in the null-space $A w=A \bar{x}-A \underline{x}=\bar{s}-\underline{s}=0$. This contradicts the assumption $\underline{s} \neq \bar{s}$. The convex function $Q(s)$ is therefore constance-free.

Conversely, suppose $c(x)=Q(A x)$ with $Q(s)$ convex and constance-free. Suppose further that points $\underline{x} \neq \bar{x}$ are endpoints of a line segment $S$ on which $c(x)$ has constant values. For $0 \leq \theta \leq 1$,

$$
c[\underline{x}+\theta(\bar{x}-\underline{x})]=Q[A \underline{x}+\theta(A \bar{x}-A \underline{x})]=Q[\underline{s}+\theta(\bar{s}-\underline{s})],
$$

where $\underline{s}=A \underline{x}, \bar{s}=A \bar{x}$. If $\underline{s} \neq \bar{s}$, the function $Q(s)$ would be constant on the line segment $A S$ in violation of the assumption that $Q(s)$ is constance-free. Thus $\underline{s}=\bar{s}$. This implies $A w=0$ for the direction $w=\bar{x}-\underline{x}$, and

$$
c(x+\theta w)=Q(A x+\theta A w)=Q(A x)=c(x)
$$

for any $x \in K$ and $\theta$.

\section{Faithfully Convex Functions}

Linear and convex quadratic functions, as well as convex analytic functions defined on the entire $R^{n}$, are weakly analytic (2.1) besides being convex. In fact, they satisfy the following stronger condition. 

(3.1) Definition: A function $c(x)$ defined on $C \subseteq R^{n}$ is

$$
\text { "faithfully convex" [5], [7] }
$$

if for any line $L$ and any line segment $S=[\underline{x}, \bar{x}] \subset L, \underline{x} \neq \bar{x}$, the following holds: if the function $c(x)$ is linear on the segment $S$, then it is defined and linear on the entire line $L$.

Again there is a companion concept of "faithfully concave". Again we will consider only the convex case, the concave case being entirely analogous.

Faithfully convex functions are plainly weakly analytic. On the other hand, it is easy to construct convex functions which are weakly analytic but not faithfully convex such as, for instance, the absolute value function $|x|$ on $R$.

For faithfully convex functions, we have in analogy to (2.3):

(3.2) If a convex function $c(x)$ defined on an open convex set $K$ is faithfully convex (3.1) the following holds: if $c(x)$ is linear on some line segment $S=[\underline{x}, \bar{x}], \underline{x} \neq \bar{x}$, then every line $L$ parallel to $S$ either lies entirely outside $K$ or is fully contained by it. On every such line contained in $K$, the function $c(x)$ is linear with the same slope. More precisely, if $x^{\prime}+\theta w, \theta \in R$, describes a line $L \subset K$ parallel to $S$, then there exists a slope $m(w)$ such that for all $x \in K$.

$$
c(x+\theta w)=c(x)+m(w) \theta
$$

Proof: Suppose the faithfully convex function $c(x)$ is linear on a line segment $S$. Then there exists a linear function $\ell(x)$ that coincides with $c(x)$ not just on $S$ but along the entire line $L \supset S$. The convex function $c(x)-\ell(x)$ is therefore constant along line $L$, and by (2.2), defined and constant on all parallel lines that meet $K$. Then $c(x)=[c(x)-\ell(x)]+\ell(x)$ is linear with identical slopes on all those lines.

It follows again that faithfully convex functions are the direct sums of linear and strictly convex functions. 


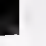

.
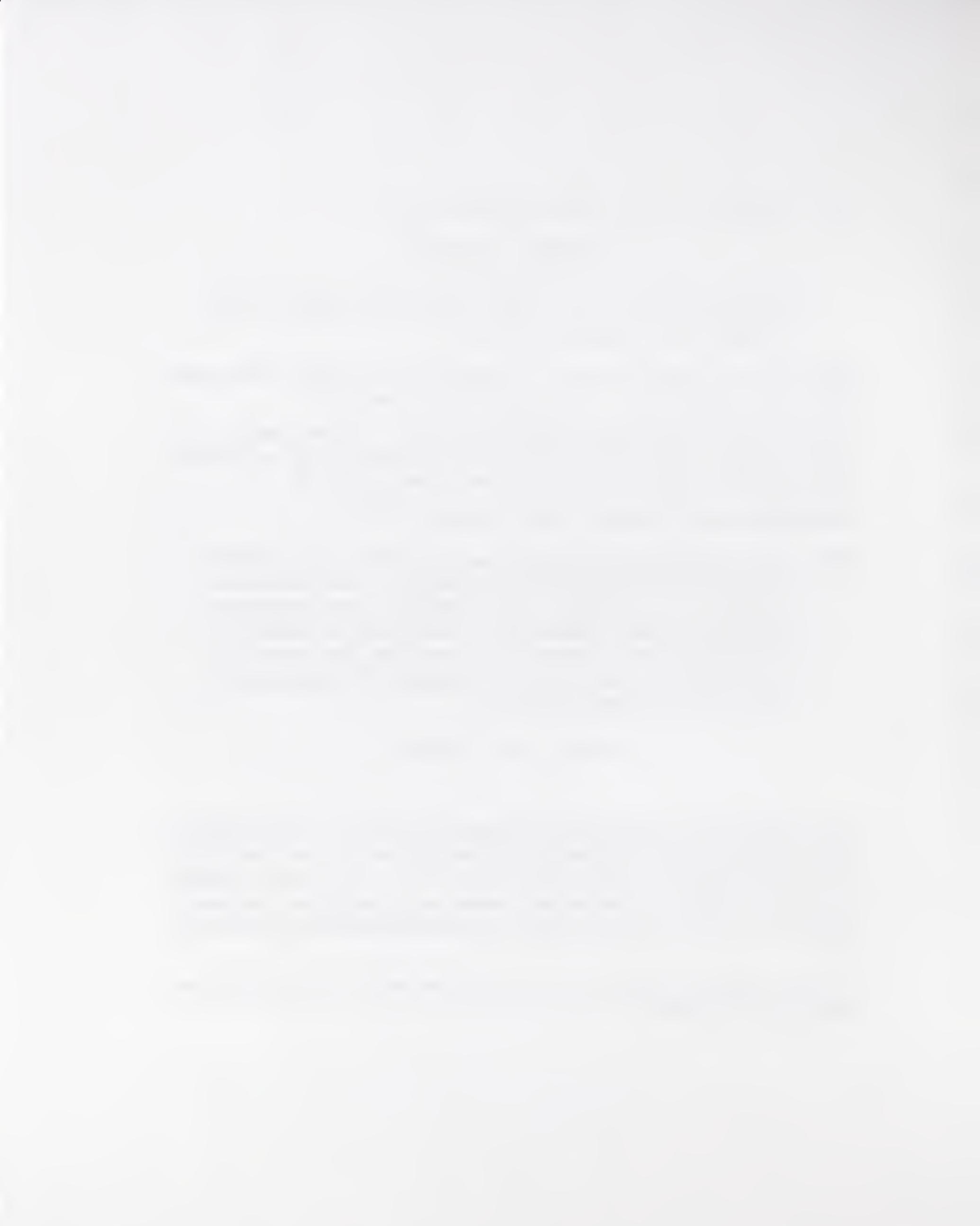
(3.3) Theorem: $A$ convex function $c(x)$ on an open convex set $K \subseteq R^{n}$ is

$$
\text { faithfully convex, }
$$

if and only if it is of the form

$$
c(x)=a^{T} x+Q(A x)
$$

with $A$ an $\ell \times n$-matrix and $Q(s), s \in A K \subseteq R^{\ell}$, a strictly convex function.

Proof: Suppose the function $c(x)$ is faithfully convex, and let $D$ be the set of all directions $w$ in which $c(x)$ is linear along a line. The key observation here is again that $D$ is a linear subspace of $R^{n}$. Again multiples of elements $u \in D$ and $v \in D$ are clearly also in $\mathrm{D}$, so that only $u+v \in D$ remains to be verified. Indeed, it suffices to show that $w=\lambda u+\mu v, \lambda, \mu \geq 0, \lambda+\mu=1$ is also in $D$. By convexity,

$$
c[x+\theta(\lambda u+\mu v)]=c[\lambda(x+\theta u)+\mu(x+\theta v)] \operatorname{leq} \lambda c(x+\theta u)+\mu c(x+\theta v),
$$

for any $x \in K$. By (3.2), the expressions $c(x+\theta u)$ and $c(x+\theta v$ represent linear functions in $\theta$. Hence there exist numbers $m(u), m(v)$ such that $c(x+\theta u)=$ $c(x)+m(u) \theta, c(x+\theta v)=c(x)+m(v) \theta$. Thus

$$
c[x+\theta(\lambda u+\mu v)] \leq c(x)+[\lambda m(u)+\mu m(v)] \theta .
$$

Actually, equation holds in the above inequality, because the convex function of $\theta \in R$ to the left is bounded above by the linear function of $\theta \in R$ to the right. The function $c[x+\theta(\lambda u+\mu v)]$ is therefore linear, too, with the same slope and intercept. Thus $w=\lambda u+\mu v \in D$, so that $D$ is indeed a linear subspace of $R^{n}$. Again we denote by $D^{\perp}$ the orthogonal complement to $D$. Also note that

$$
m(w)=m(\lambda u+\mu v)=\lambda m(u)+\mu m(v),
$$

in other words, $m(w)$ is a linear function for $w \in D$. Consequently, there exist vectors $b \in R^{n}$ such that $m(w)=b^{T} w$ for $w \in D$. Any such vector $b$ can be (uniquely) represented in the form $b=a+t$ with $a \in D$ and $t \in D^{\perp}$. Since $t^{T} w=0$ for all $w \in D$, it follows that $b^{T} w=a^{T} w+t^{T} w=a^{T} w=m(w)$ for $w \in D$. In particular,

$$
c(x+w)=c(x)+a^{T} w \text { for } x \in K \text { and } w \in D .
$$

As a linear subspace, $D$ can, as before, be described as the null-space of an $\ell \times n$-matrix $A$ of full rank $\ell: D=\{w: A w=0\}$. And, as in the previous 
section, we write $x=w+\hat{x}$ with $w \in D$ and $\hat{x}=A^{T}\left(A A^{T}\right)^{-1} A x \in D^{\perp}$. Since $a \in D$ and $\hat{x} \in D^{\perp}$, we have $a^{T} x=a^{T}(w+\hat{x})=a^{T} w$. Thus by (3.4),

$$
c(x)=c(w+\hat{x})=c(\hat{x})+a^{T} w=a^{T} x+c\left[A^{T}\left(A A^{T}\right)^{-1} A x\right]=a^{T} x+Q(A x) .
$$

We have to show that the plainly convex function

$$
Q(s)=c\left[A^{T}\left(A A^{T}\right)^{-1} s\right], s \in A K,
$$

is strictly convex. Assume to the contrary that $Q(s)$ is linear on some line segment $S=[\underline{s}, \bar{s}], \underline{s} \neq \bar{s}$. Then $\underline{x}=A^{T}\left(A A^{T}\right)^{-1} \underline{s}$ and $\bar{x}=A^{T}\left(A A^{T}\right)^{-1} \bar{s}$ are points in $R^{n}$ with $\underline{s}=A \underline{x}$ and $\bar{s}=A \bar{x}$. It follows that the points $\underline{x}, \bar{x}$ bracket a line segment $S$ for which $c(x)$ is linear. By weak linearity, $c(x)$ is then linear everywhere in direction $w=\bar{x}-\underline{x}$, which thus must lie in the null-space $A w=A \bar{x}-A \underline{x}=$ $\bar{s}-\underline{s}=0$. This contradicts the assumption $\underline{s} \neq \bar{s}$. The convex function $Q(s)$ is thus strictly convex.

Conversely, suppose $c(x)=a^{T} x+Q(A x)$ with $Q(s)$ strictly convex. Suppose further that points $\underline{x} \neq \bar{x}$ are endpoints of a line segment $S$ on which $c(x)$ is linear. Then $Q(A x)$ is also linear on $S$. For $0 \leq \theta \leq 1$,

$$
Q[A \underline{x}+\theta(A \bar{x}-A \underline{x})]=Q[\underline{s}+\theta(\bar{s}-\underline{s})],
$$

where $\underline{s}=A \underline{x}, \bar{s}=A \bar{x}$. Since $Q(s)$ is strictly convex, $\underline{s}=\bar{s}$. This implies $A w=0$ for the direction $w=\bar{x}-\underline{x}$. It follows that

$$
Q(A \underline{x}+\theta A w)=Q(A \underline{x})
$$

and

$$
c(\underline{x}+\theta w)=a^{T} \underline{x}+\theta a^{T} w .
$$

Thus $c(x)$ is linear on the entire line through segment $S$.

Weak analyticity is not additive, not even for convex functions. For instance, $|x-1|+|x+1|$ is the sum of weakly analytic convex functions but itself is not weakly analytic. By contrast, faithfully convex functions are additive. Thus functions of the form

$$
c(x)=a^{T} x+Q_{1}\left(A_{1} x\right)+\ldots+Q_{k}\left(A_{k} x\right),
$$

with strictly convex functions $Q_{1}, \ldots, Q_{k}$, are also of the form $a^{T} x+Q(A x)$ since additivity holds: $Q_{1}\left(A_{1} x\right)+Q_{2}\left(A_{2} x\right)=Q(A x)$ with $Q=Q_{1} \oplus Q_{2}$ the - strictly convex - direct sum of two strictly convex functions and

$$
A=\left(\begin{array}{c}
A_{1} \\
A_{2}
\end{array}\right)
$$





\section{Self-Concordant Functions}

The purpose of this section is to establish the faithful convexity of self-concordant functions in the case that their region of definition is an entire euclidean space $R^{n}$. So for any function $c(x)$ defined on the entire $R^{n}$ and any straight line

$$
L=\left\{x_{0}+h t: t \in R, x_{0} \in R^{n},\|h\|=1\right\} \in R^{n},
$$

we define the

\section{"restriction"}

of $c(x)$ to $L$ as the function $f(t)=c\left(x_{0}+h t\right)$ of one variable. It is plain that

(4.2) a function $c(x), x \in R^{n}$, is faithfully convex (3.1)) if every restriction (4.1) of $c(x)$ to a line $L \in R^{n}$ is faithfully convex as a function of one variable.

As a result, we turn our attention to functions $f(t)$ of a single variable $t \in R$. In particular, such a function $f(t)$ is faithfully convex if it is either strictly convex or linear.

For the purpose of the following analysis, we say that a function $g(t)$ of one real variable $t \in R$ has a

$$
\text { "value - bounded" }
$$

derivative in an interval $[a, b], b>a, a, b \in R$, if there exists $M=M(a, b)>0$ such that

$$
\left|g^{\prime}(t)\right| \leq M g(t) \text { for } t \in[a, b] .
$$


. 
(4.4) Lemma: Suppose

$$
\begin{aligned}
\text { (i) } & g(t) \geq 0 \text { for } t \geq 0 \\
\text { (ii) } & g(0)=0 \\
\text { (iii) } & g^{\prime}(t) \text { exists for } t \geq 0 \\
\text { (iv) } & g^{\prime}(0)=0
\end{aligned}
$$

Suppose further that the function $g(t)$ has a value-bounded derivative (4.3) with factor $M$ for some interval $[0, u], u>0$. Define $v>0$ by

$$
v=\min \left\{\frac{1}{M}, u\right\}
$$

Then

$$
g(t)=0 \text { for } t \in[0, v]
$$

Proof: Consider the function

$$
s(t)=\left\{\begin{array}{l}
g(t) / t \quad \text { for } t \in(0, v] \\
0 \text { for } t=0
\end{array}\right.
$$

As

$$
\frac{g(t)}{t} \rightarrow g^{\prime}(0)=0
$$

the function $s(t)$ is continuous in the closed interval $[0, v]$ and, consequently, assumes its maximum for some argument $t^{(0)}$ in that interval. Note that

$$
s\left(t^{(0)}\right)=0 \text { implies } g(t)=0 \text { for } t \in[0, v] .
$$

For that reason, we may also assume that

$$
t^{(0)}>0
$$

since $t^{(0)}=0$ implies $s\left(t^{0}\right)=0$, so that $s(t)$ vanishes in $[0, v]$ and a maximizer could be chosen anywhere in the interior of that interval instead.

By the mean value theorem, there exists

$$
\hat{t}, 0<\hat{t}<t^{(0)} \quad \text { with } g\left(t^{(0)}\right)=t^{(0)} g^{\prime}(\hat{t}) .
$$


Thus, since $t^{(0)}$ maximizes the function $s(t)$ in $[0, v]$, and using the fact that the derivative $g^{\prime}(t)$ is value-bounded (4.3), we find

$$
0 \leq s(\hat{t}) \leq s\left(t^{(0)}\right)=\frac{g\left(t^{(0)}\right)}{t^{(0)}}=g^{\prime}(\hat{t}) \leq M g(\hat{t})=M \hat{t} s(\hat{t}) .
$$

As $0<\hat{t}<t^{(0)} \leq v \leq 1 / M$ by the definition of $v$ in Lemma (4.4),

$$
M \hat{t}<1 .
$$

The inequalities (4.6) may, therefore, hold only if $s(\hat{t})=0$ and, consequently, both $g^{\prime}(\hat{t})$ and $s\left(t^{(0)}\right)=0$. By (4.5), this implies $g(t)=0$ for $t \in[0, v]$, which was to be shown. (That $s(\hat{t})=0$ implies $g^{\prime}(\hat{t})=0$ and, therefore, $s\left(t^{(0)}\right)=0$, follows also from the fact that $g(\hat{t})=\hat{t} s(\hat{t})=0$ is assuming a minimum.)

(4.7) Proposition: A convex function $f(t)$, defined everywhere on the real number line $R$, is faithfully convex if its second derivative is valuebounded (4.3) in every interval $[a, b], b>a, a, b \in R$.

Proof. If $f(t)$ is strictly convex, nothing needs to be shown. Suppose then that $f(t)$ is linear in a closed interval of positive length. Without loss of generality, we assume that the interval has a right end of zero. The function $g(t)=f^{\prime \prime}(t)$ vanishes in the interval, and satisfies conditions (i),...,(iii) for Lemma (4.4). Condition (iv) is met because $g(t) / t$ has a left limit of zero at $t=0$ so that $g^{\prime}(0)=0$ by differentiability (continuity of $g^{\prime}(t)$ is not used).

By hypothesis, $g(t)$ is value-bounded in an interval $[0, u], u>0$. By Lemma (4.4), there exists $v>0$ such that

$$
g(t)=0 \text { for } t \in[0, v] .
$$

Any zero interval of $g(t)$ thus can be extended to the right. Any upper end of those extensions is, by continuity, itself an upper end to a zero interval, and therefore extendible, contradicting that it is a bound to all previously found upper ends. This rules out a finite upper end to any zero interval.

The analogous argument applied to $\tilde{g}(t)=g(-t)$, and taking into account

$$
\tilde{g}^{\prime}(t)=-g^{\prime}(-t) \leq-\left|g^{\prime}(-t)\right|,
$$

shows that a lower end to a zero interval cannot exist, either. Thus $g(t)$ vanishes for all real $t$, and $f(t)$ is a linear function. 
(4.9) Definition: A convex function $c(x)$ defined for $x \in R^{n}$ is

$$
\text { "self - concordant" [4] }
$$

if every one of its restrictions $f(t)$ (see (4.1)) has a continuous third derivative $f^{\prime \prime \prime}(t)$ satisfying

$$
\left|f^{\prime \prime \prime}(t)\right| \leq 2\left(f^{\prime \prime}(t)^{3 / 2}\right)
$$

Compare also [2]. We proceed to show that self-concordance implies faithful convexity.

(4.10) Theorem: Self-concordant functions $c(x)$ are faithfully convex if defined on an entire euclidean space $R^{n}$.

Proof: In view of (4.2), we have only to show that the restrictions $f(t), t \in R$, of $c(x)$ are faithfully convex as a consequence of the self-concordance inequality in Definition (4.9). Again, $g(t)=f^{\prime \prime}(t) \geq 0$ since $f(t)$ is convex, and the aforementioned inequality becomes

$$
\left|g^{\prime}(t)\right| \leq 2(g(t))^{3 / 2} \text {. }
$$

By continuity, $2 g(t)^{1 / 2}$ has an upper bound $M=M(a, b)>0$ in any interval $[a, b], b>a, a, b \in R$. In other words, $g(t)$ has a value-bounded derivative in any such interval, and $f(t)$ is faithfully convex by Proposition (4.7).

\section{References}

[1] Bank, B., Guddat, J., Klatte, D., Kummer, B., and Tammer, K. Non-Linear Parametric Optimization. Akademie Verlag, Berlin, 1982.

[2] JARRE, F. Interior point methods for classes of convex programs. In Interior Point Methods of Mathematical Programming, T. Terlaky, Ed. Kluwer Academic Publishers, Boston, 1996, pp. 255-296.

[3] MCCormick, G. P., AND Witzgall, C. Logarithmic SUMT limits in convex programming. (in preparation). 


\section{.}


[4] Nesterov, Y. E., AND Nemirovskil, A. S. Interior-point polynomial algorithms in convex programming, vol. 13 of SIAM Studies in Applied Mathematics. Society for industrial and Applied Mathematics (SIAM), 1994.

[5] Rockafellar, R. T. Some convex programs whose duals are linearly constrained. In Nonlinear Programming, O. L. Mangasarian and K. Ritter, Eds. Academic Press, New York, 1970, pp. 293-322.

[6] Rockafellar, R. T. Convex Analysis. Princeton University Press, Princeton, New Jersey, 1970, 2-nd printing, 1997.

[7] RockafellaR, R. T. Ordinary convex programs without a duality gap. $J$. Optim. Theory Appl. 7 (1971), 143-148.

[8] Stoer, J., And Witzgall, C. Convexity and Optimization in Finite Dimensions, vol. 163 of Die Grundlehren der Mathematischen Wissenschaften. Springer Verlag, Berlin, 1970. 


